\title{
Development status of the LAUE project
}

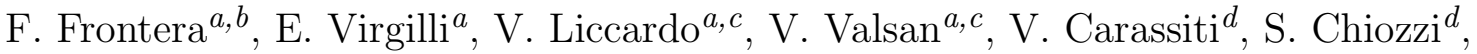 \\ F. Evangelisti ${ }^{d}$, S. Squerzanti ${ }^{d}$, M. Statera ${ }^{a}$, V. Guidi ${ }^{a}$, C. Ferrari ${ }^{e}$, R. A. Zappettini ${ }^{e}$,

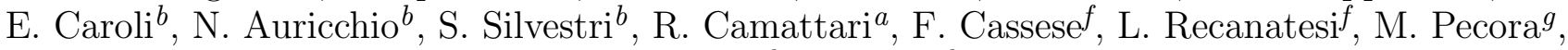 \\ S. Mottini ${ }^{h}$, B. Negri ${ }^{k}$ \\ ${ }^{a}$ Physics Department, University of Ferrara - Via Saragat, 1, 44100 Ferrara - Italy; \\ ${ }^{b}$ INAF-IASF Bologna, via P. Gobetti 101, Bologna - Italy; \\ ${ }^{c}$ Université de Nice Sophia Antipolis, Nice, Cedex 2, Grand Chateau Parc Valrose - France; \\ ${ }^{d}$ INFN, Sezione di Ferrara, via Saragat 1, 44100 Ferrara - Italy; \\ e CNR, IMEM, Parco Area delle Scienze 37/A - 43124 Parma - Italy; \\ ${ }^{f}$ DTM, Modena, Via Tacito, I-41100 Modena - Italy; \\ $g$ Thales Alenia Space-Italy, Milan - Italy; \\ $h$ Thales Alenia Space-Italy, Turin - Italy; \\ ${ }^{k}$ ASI, Agenzia Spaziale Italiana, Viale Liegi 26, I-00198 Roma - Italy.
}

\begin{abstract}
We present the status of LAUE, a project supported by the Italian Space Agency (ASI), and devoted to develop Laue lenses with long focal length (from 10-15 meters up to 100 meters), for hard X-/soft gamma-ray astronomy (80-600 keV). Thanks to their focusing capability, the design goal is to improve the sensitivity of the current instrumention in the above energy band by 2 orders of magnitude, down to a few times $10^{-8}$ photons $/\left(\mathrm{cm}^{2} \mathrm{~s} \mathrm{keV}\right)$.
\end{abstract}

Keywords: Laue lenses, focusing telescopes, gamma-rays, Astrophysics.

\section{INTRODUCTION}

Many astrophysical issues are expected to be solved with focusing telescopes that cover the soft gamma-ray band (beyond $80 / 100 \mathrm{keV}$ ), if they can reach a sensitivity a factor from 10 to 100 better than that of the current instrumentation (e.g., IBIS instrument aboard INTEGRAL ${ }^{11}$ ). A discussion of the achievable scientific obiectives has been extensively discussed in more occasions (see, e.g., Ref. [2]).

Among the many astrophysical issues that could be settled, we like to mention one of the most elusive questions since many years: the origin of positrons in the Galactic Center (CG) region. A SPI/INTEGRAL allsky map of the galactic $\mathrm{e}^{-} / \mathrm{e}^{+}$annihilation radiation at $511 \mathrm{keV}$ shows an asymmetric distribution around the $\mathrm{GC}^{3}$. The authors of the paper give a possible interpretation in terms of superposition of the discrete positron annihilation radiation sources. These sources should belong of a special class of Low Mass X-ray Binaries (called hard LMXBs) located in the CG region, that are strong emitters of hard X-rays $(>20 \mathrm{keV})$. However other possible interpretations have been advanced by other authors, such as the presence of an extended region of antimatter or dark matter, or the existence of a source of radioactive elements, like ${ }^{26} \mathrm{Al},{ }^{56} \mathrm{Co},{ }^{44} \mathrm{Ti}$, or the presence of a gamma-ray source (e.g., gamma-ray pulsar). Only much more sensitive observations can settle the origin of the annihilation line from the GC region.

We have already reported on the LAUE project last year ${ }^{[4}$. It is the follow-up of an initial development project (HAXTEL), also supported by ASI, that allowed to implement a concept of assembling technique for Laue lenses ${ }^{5 / 6}$, to significantly shorten the time needed to build a lens. On the basis of the tests performed on two lens prototypes built according to the HAXTEL technology, we found that the implemented technique is satisfactory only for short focal-length Laue lenses $(<10 \mathrm{~m})$.

Given the sensitivity and the energy broad-band to be covered with the first generation of these conceptually new focusing telescopes, focal lengths longer than 10 meters are required. These reasons led the birth of a new project named LAUE, that was preliminary discussed in Ref. 4]. Now the project is definitive and it can be better described.

E-mail to: frontera@fe.infn.it 


\section{THE LAUE PROJECT: A FOCUSING LENS FOR SOFT GAMMA-RAYS}

The sketch of a Laue Lens for space astrophysics and the required orientation of the crystals in the lens are shown in Figure 1] An extensive review on Laue lenses can be found in Ref. [2].
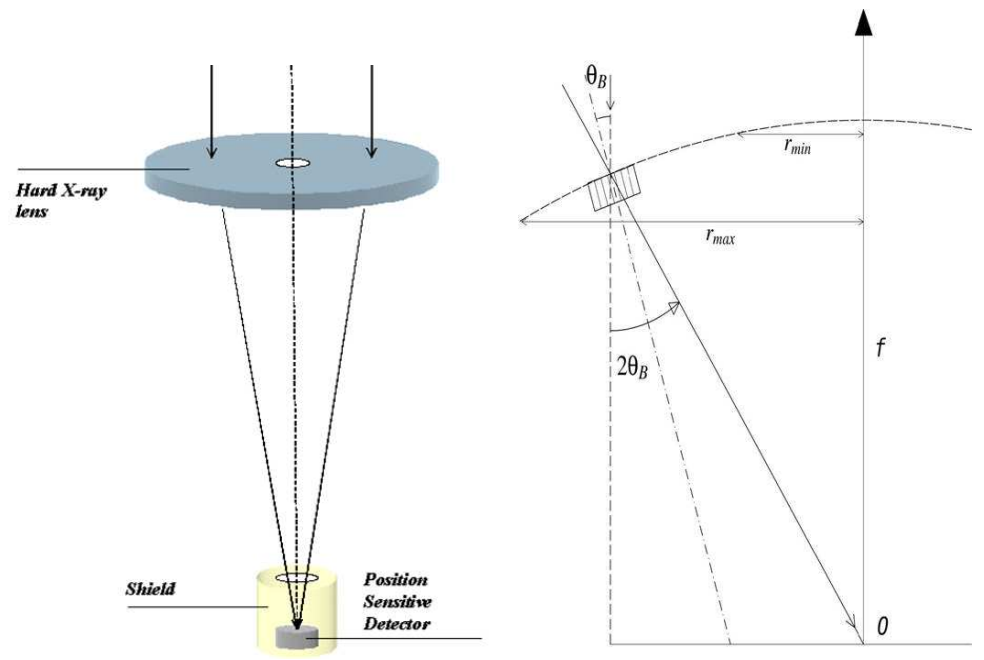

Figure 1. Left panel: sketch of a Laue lens. Right panel: orientation of crystals in a Laue lens.

The main goal of the LAUE project is to develop an advanced technology for building a sensitive Laue lens with broad energy band (>80/100 keV) and long focal length (up to $100 \mathrm{~m}$ ) for space astrophysics but the project also faces a still long standing and difficult issue: the development of suitable crystals for gamma-ray lenses.

From the experience gained with the HAXTEL project, we have adopted the following approach for positioning the crystals on a lens frame. Under the control of a gamma-ray beam, each crystal tile is properly positioned when it focuses the reflected photon beam to the lens focus. Once the correct orientation is found, each crystal tile is fixed to the lens frame, which is kept fixed during the lens assembling process. This implies that the beam has to be continuously translated to assemble the crystal tiles. This approach is expected to minimize the orientation errors. Given the large size (diameter larger than $1 \mathrm{~m}$ ) of space lenses, the only feasible way to build such lenses, is that of subdividing them in a number of lens petals. The feasibility study of a similar approach for building a space lens is being performed within the LAUE project (see Figure 2). One of the lens petals, with $20 \mathrm{~m}$ focal length and filled with about 300 crystals tiles, will be built as a result of the LAUE project.

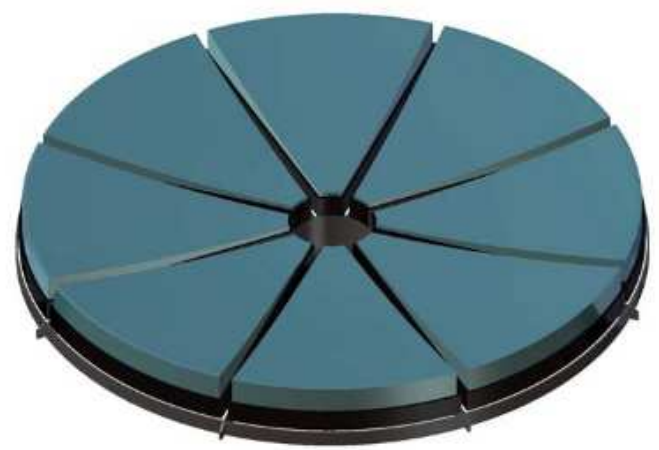

Figure 2. View of a space lens made of petals. The building of one of these petals is one of the goals of the LAUE project. 


\section{APPARATUS FOR LENS ASSEMBLING}

A lens assembling apparatus is being installed in a tunnel of the LARge Italian X-ray Facility (LARIX) of the Physics Department of the University of Ferrara. Its drawing is shown in Figure 3, The two enters of the tunnel are properly shielded from the $\mathrm{X} /$ gamma radiation. On one side, close to the B section of the LARIX facility, an L-shaped lead wall surrounding the source is set. On the opposite side, beyond the detectors, a motorized lead door $65 \mathrm{~mm}$ thick has been already installed. This guarantees a free use of the laboratories located outside the tunnel: LARIX A at the beginning of the tunnel, and LARIX B at its end. The former section will be used as remote control room for managing the LAUE apparatus and the petal lens assembling.

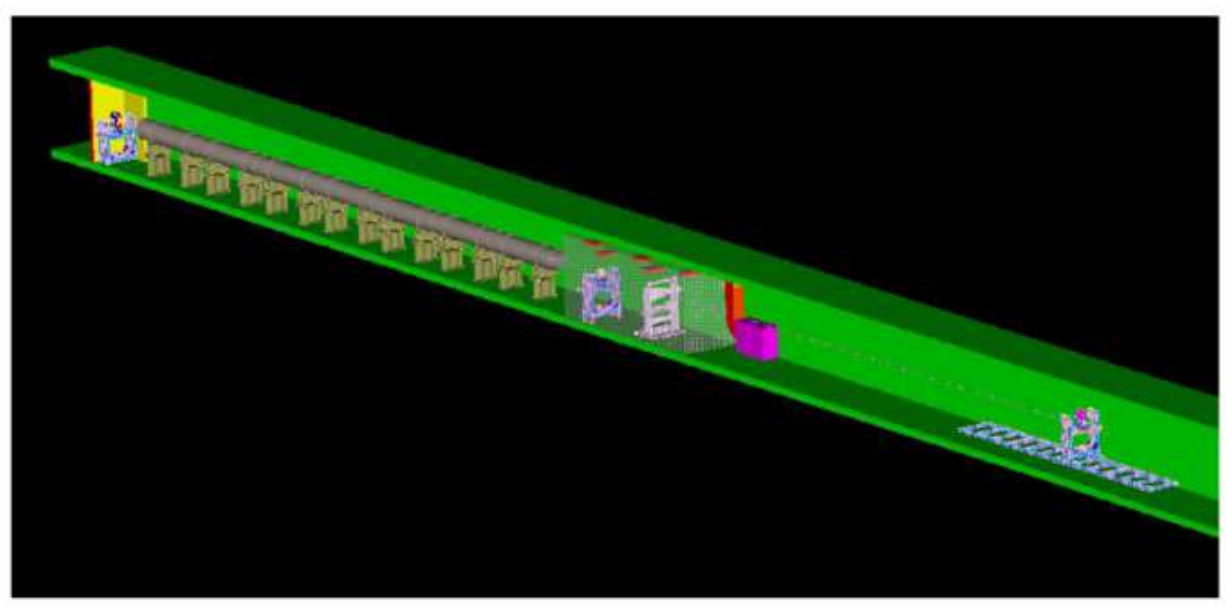

Figure 3. Drawing of the LAUE apparatus located in the tunnel of the LARIX facility. It is visible the X-.ray source surrounded by the protection lead wall (on the left), the beam line, the clean room with controled temperature and environment in which the lens will be assembled (at the center), and the focal plane detector holder mounted on a rail.

The main subsystems of the apparatus include a gamma-ray generator, a beam-line, a clean room, with temperature and humidity control, in which the lens petal will be assembled, and a focal plane detection system, with its holder mounted on a rail that can be moved back and forth.

The clean room hosts the following subsystems: 1) a slit collimator for controlling the size of the gamma-ray beam impinging on the crystal tile, 2) the lens frame, 3) a multi-axis positioning system for correctly positioning and orienting crystal tiles on the lens, 4) an automated system for fixing crystal tiles to the frame. In the following subsections details about the main apparatus components are presented.

\subsection{Gamma-ray source system}

The gamma-ray source system includes a portable betatron and an hard $\mathrm{X}$-ray generator. The betatron has a maximum electron energy that can be regulated between 1 and $2.5 \mathrm{MeV}$, with a Tungsten target on which the accelerated electrons impinge for producing bremsstrahlung radiation. The maximum betatron power is $310 \mathrm{~W}$, with a gamma-ray focal spot less than $0.2 \times 3 \mathrm{~mm}$.

The hard X-ray generator has a maximum voltage of $320 \mathrm{kV}$, an X-ray tube with a tungsten anode and a fine focus of $0.2 \mathrm{~mm}$ radius. The maximum releasable power is $\sim 1800 \mathrm{~W}$. The expected photon spectrum from the hard X-ray generator and for the betatron are shown in Figure 4 .

The X-ray tube, providing a higher fluence than that of the betatron, will be used for the lens building phase, given that a higher fluence imply a shorter integration time for the tiles allignment stage. On the other hand, the betatron source can be used for the performence tests and, more important, will allow to build Laue lenses focusing higher energies than that designed lens petal.

The gamma-ray source holder can be remotely translated along two perpendicular axes y and z (vertical) (the $\mathrm{x}$ axis is directed along the tunnel direction) and can be tilted around the $\mathrm{z}$ axis. 

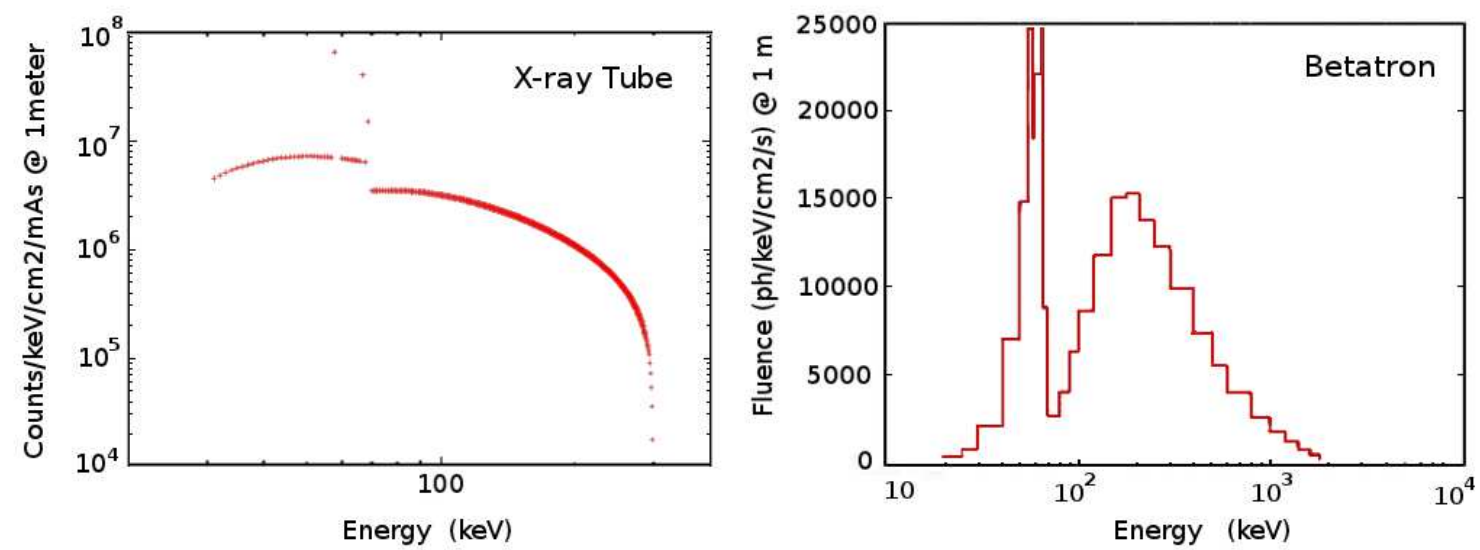

Figure 4. Expected photon spectrum (in photons $\mathrm{keV}^{-1} \mathrm{~cm}^{-2} \mathrm{~s}^{-1}$ ) at $1 \mathrm{~m}$ distance of the $\mathrm{X}-$ ray tube (left) and from the betatron (right).

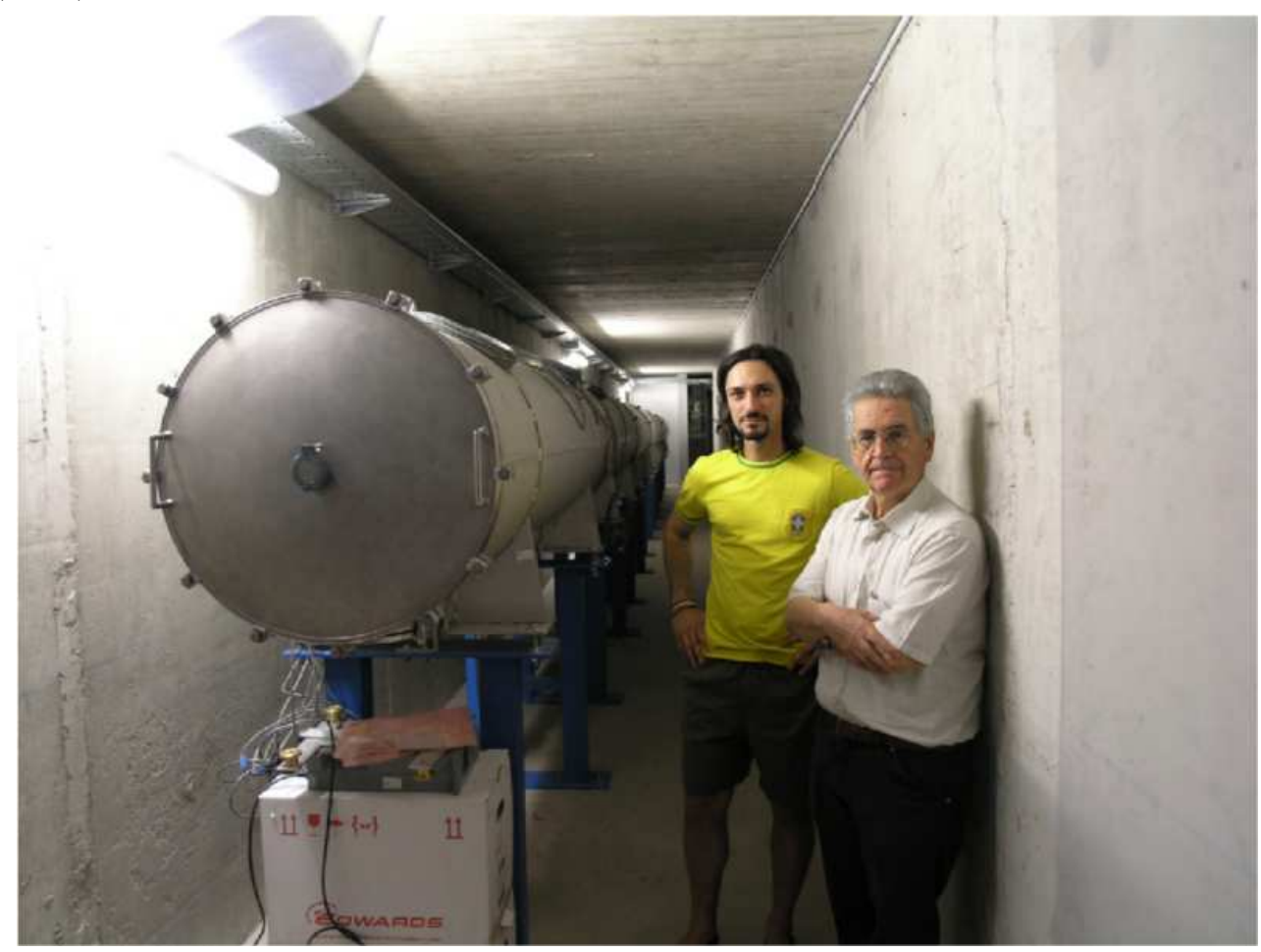

Figure 5. A view of the $21 \mathrm{~m}$ beam-line installed in the LARIX tunnel.

\subsection{Beam-line}

The photons coming from the gamma-ray source, after an initial collimation, enter in a $21 \mathrm{~m}$ long beam-line. It is made of seven $3 \mathrm{~m}$ long tubes of stainless steel with an internal diameter of $60 \mathrm{~cm}$. A set of vacuum pumps guarantees a vacuum of 1 mbar inside the tubes. The $\mathrm{X}$-ray entrance and exit windows of the beam-line are made of carbon fiber $3 \mathrm{~mm}$ thick. The prospect is to extend the beam-line will be extended up to $70 \mathrm{~m}$. A view of the already installed beam-line is shown in Figure 5 . 


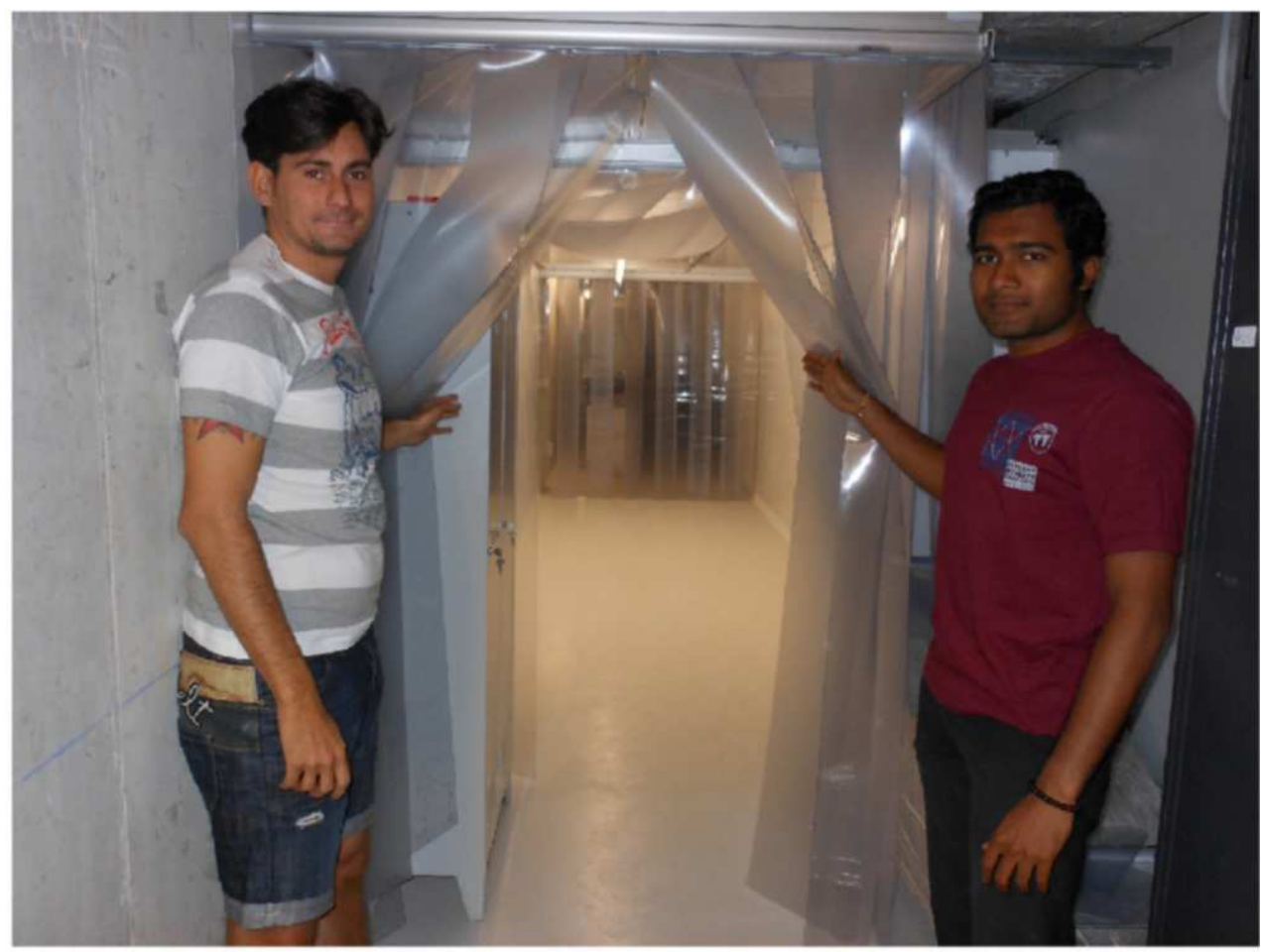

Figure 6. A view of the entrance in the clean-room already installed in the LARIX tunnel.

\subsection{Clean-room and the main apparatus components inside}

Once the gamma-ray beam exits from the beam-line, it enters in a clean-room (class better than $10^{5}$, US FED STD 209E Cleanroom Standards). The clean-room is endowed with a thermal control (within $1{ }^{\circ} \mathrm{C}$ accuracy) and an hygrometric control (relative humidity $\Phi=60 \%$ within an error of $10 \%$ ). A view of the entrance in the clean-room is shown in Figure 6. The main components inside the clean-room are the following.

\section{- Slit collimator}

A slit collimator, made of four independent blades of Tungsten $20 \mathrm{~mm}$ thick, with variable aperture along two ortogonal directions allows to regulate, from the remote control room, the size of the gamma-ray beam coming from the beam-line. The beam size establishes the beam divergence. In addition, the slit collimator can be accurately translated along the $\mathrm{y}$ and $\mathrm{z}$ axes and rotated around the $\mathrm{z}$ and $\mathrm{x}$ axes. The collimator translation follows the source translation, in order to have the direction of the gamma-ray beam always parallel to the lens axis.

\section{- Frame of the lens petal}

The frame of the lens petal, on which the crystal tiles will be pasted, is made of carbon fiber. A view of the petal frame breadboard is shown in Figure 7

\section{- Crystal positioning system}

The positioning of each crystal tile on the petal frame is performed by using a mechanical system made of a coarse positioner and a fine positioner. The coarse positioner is made of two perpendicular translation stages, while the fine positioner, also called esapod, for its 6 degrees of freedom, allows to accurately control the crystal tilting around two perpendicular axes and its motion toward the frame. A view of the esapod under test is shown in Figure 8 


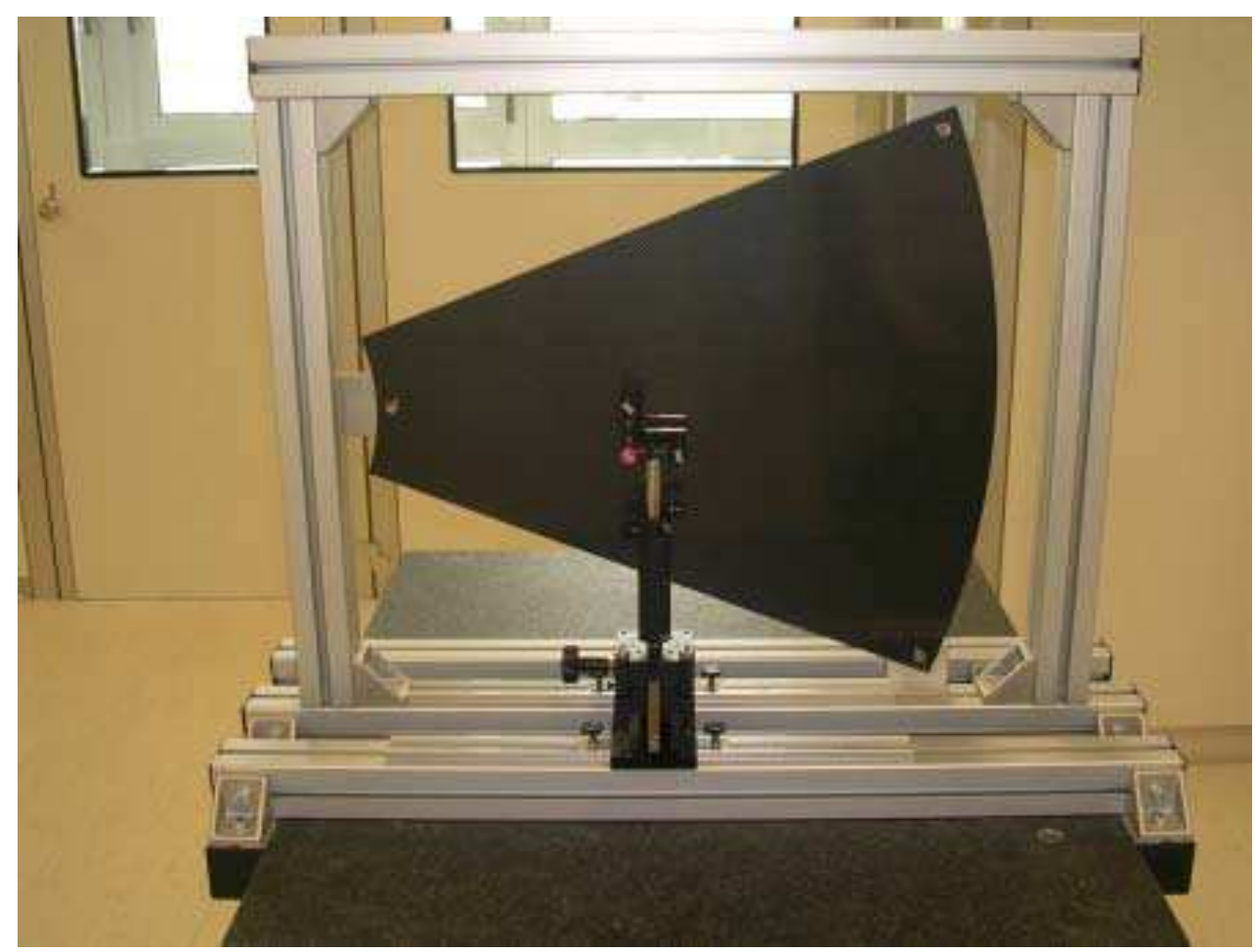

Figure 7. A view of the breadboard of the petal frame.

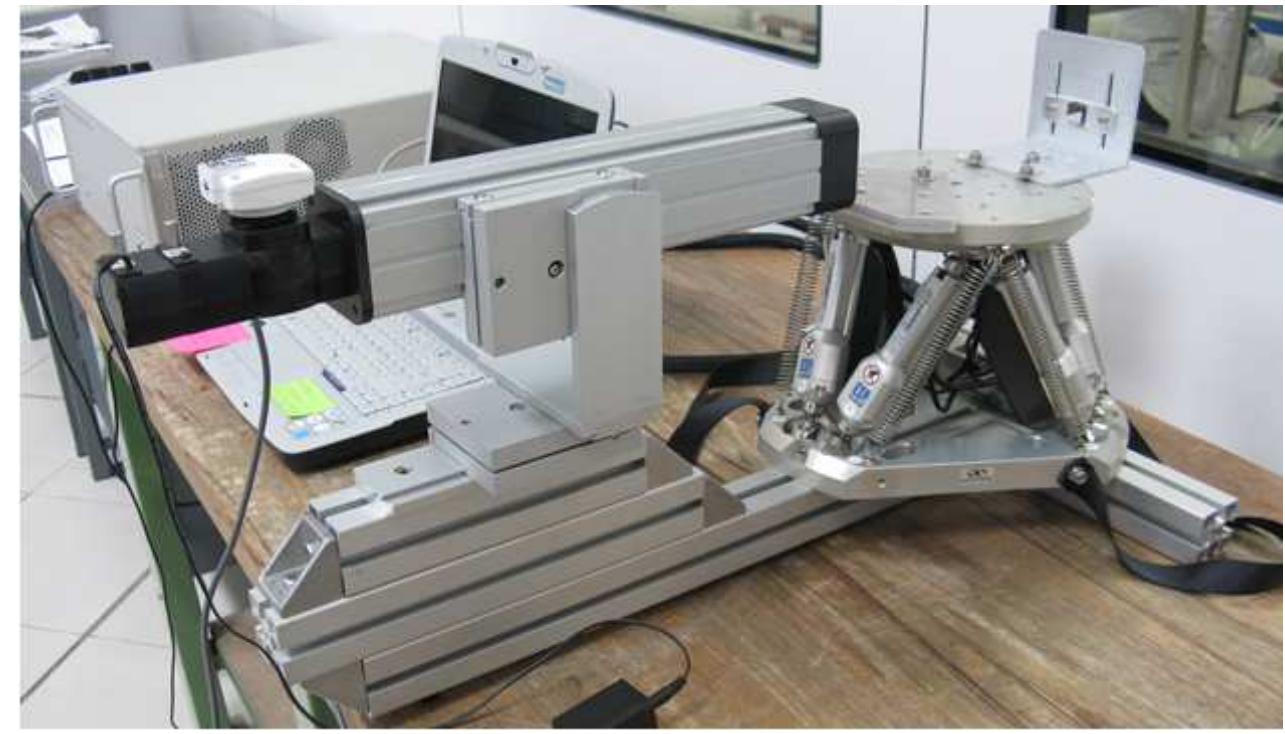

Figure 8. A view of the esapod under test.

\section{FOCAL PLANE DETECTION SYSTEM}

A gamma-ray detector system allows to establish the correct orientation of each crystal tile. It is made of two focal plane detectors: 1) an X-ray imaging detector with a useful area $20 \times 20 \mathrm{~cm}^{2}$, with a spatial resolution $200 \mu \mathrm{m}$ and an operational energy range from $20 \mathrm{keV}$ to $15 \mathrm{MeV}, 2)$ a cooled HPGe spectrometer with $2.5 \mathrm{~cm}$ diameter and an energy resolution about $500 \mathrm{eV}$ at $122 \mathrm{keV}$.

In addition to the possibility to be moved back and forth, the detector system can be translated along y and 


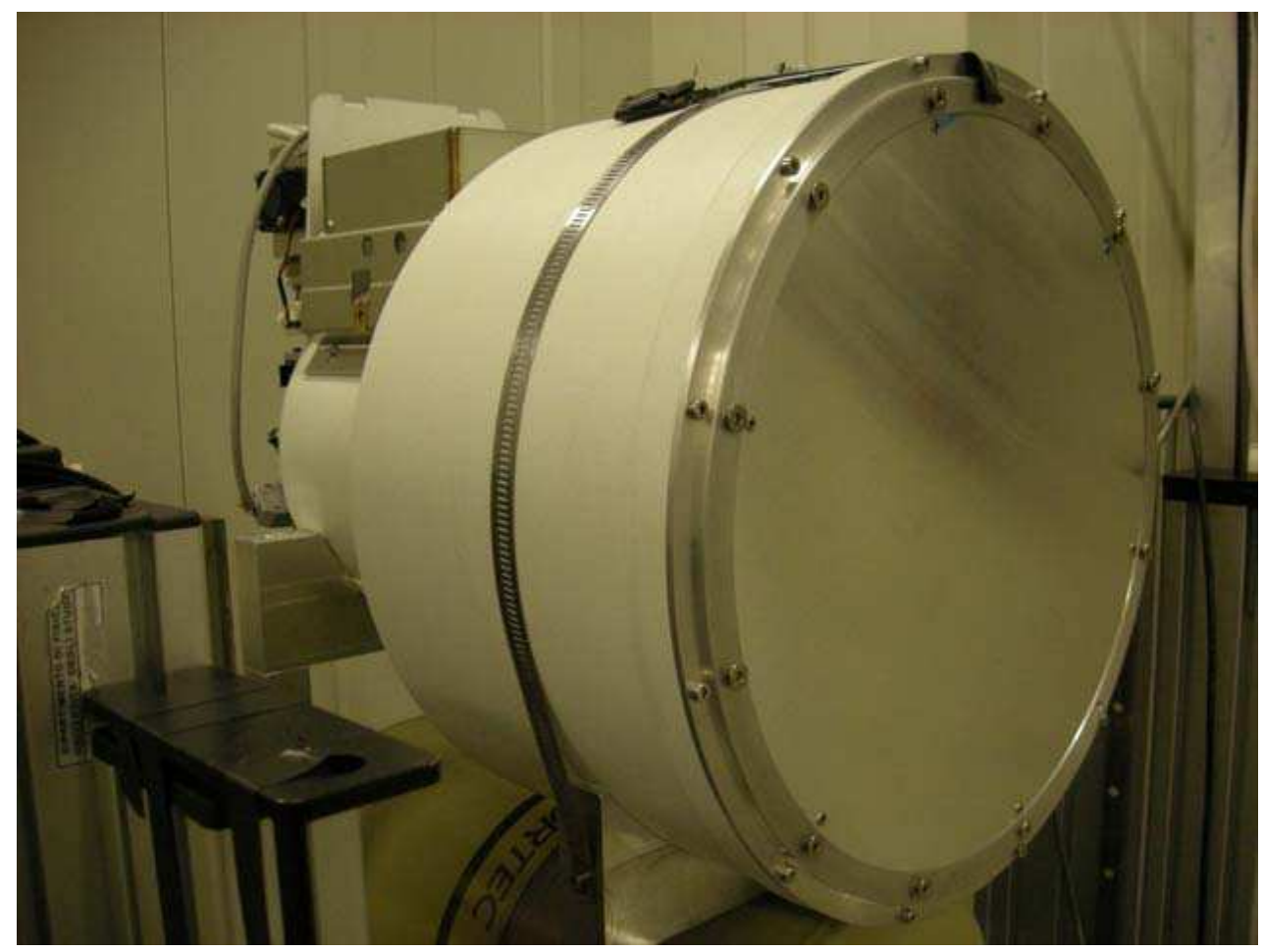

Figure 9. A view of an imaging detector similar to that used for the LAUE project.

$\mathrm{z}$ axes and can be rotated along the $\mathrm{x}$ axis. A view of the imaging detector is shown in Figure 9 .

\subsection{Managing of the lens assembling system}

As discussed above, the LAUE apparatus is located in the LARIX tunnel. It is controlled from a remote console, located in the section A of the LARIX facility that manages all the required functions for calibrating the facility and for assembling a lens petal. The main functions that can be managed from the remote console are the following:

- to set up the gamma-ray beam features (e.g., intensity, size) and translate its axis within the beam-line tube cross-section;

- to position the slit collimator such that the beam axis is kept parallel to the lens axis;

- to properly orient the crystal tile before it can fixed to the petal frame;

- to acquire spectra and images from the focal plane detectors;

- to determine the position error of the reflected beam with respect to the lens focus.

- to send the position error to the esapod for a further crystal orienting step.

\section{CRYSTALS ADOPTED FOR THE LENS PETAL}

After a development phase, the crystals that will be used for lens petal will be a combination of bent perfect crystals of $\mathrm{Ge}(111)$ and bent mosaic crystals of GaAs(111). The GaAs mosaicity is about 20 arcsec. The advantage of bent crystals is their better focusing properties (see Ref. [2]).

The crystal cross section has been chosen to be $30 \times 10 \mathrm{~mm}^{2}$, with the longer side radially placed on the lens frame. The main advantages of the rectangular shape, together with the radial disposition, concerns the focusing 
effect provided by bent crystals, which only acts in the radial direction. In such a way, a shorter tangential dimension provides a smaller defocusing factor, being proportional to the tile size. On the other hand, a bigger radial dimension allows to decrease the total number of crystals, reducing the error budget potentially caused by each crystal misalignment contribution.

The thickness $t$ of the crystal tiles is $2 \mathrm{~mm}$ for both crystal materials. The thickness value is imposed by the current status of the bending technology adopted for bending $\mathrm{Ge}^{7}$ and GaAs ${ }^{8}$. The expected performance of the adopted crystals and the experimental reflectivity results obtained from the laboratory tests performed in tha LARIX facility are discussed in two papers, one by Valsan et al .9$]$ and the other by Liccardo et al $\frac{10}{.0}$. Both will be published, along with our paper, in the same issue of the SPIE Proceedings.

\section{CONCLUSIONS}

From the already gained experience on Laue lenses (see, e.g., 5, 6]), we have started a new project, LAUE, supported by the Italian Space Agency (ASI), devoted to the development of an advanced lens assembling technology that we expect it eventually will allow to accurately build Laue lenses for space astrophysics. The expected accuracy in the lens assembling would allow to build lenses even with very long focal lengths (up to $100 \mathrm{~m}$ ), a goal never achieved so far. The LAUE apparatus, described in this paper, is being installed in the LARIX facility of the University of Ferrara. We expect in a few months to start the assembling activity of a lens petal, made of about 300 bent crystals of Ge(111) and GaAs(111). Results on the built lens petal prototype are expected to be reported in the next year SPIE plenary conference.

\section{ACKNOWLEDGMENTS}

The LAUE project is the result of big efforts made by a large number of organizations and people. We would like to thank all of them. We also acknowledge the ASI Italian Space Agency for its support to the LAUE project under contract I/068/09/0.

Vincenzo Liccardo and Vineeth Valsan are supported by the Erasmus Mundus Joint Doctorate Program by Grant Number 2010-1816 from the EACEA of the European Commission.

\section{REFERENCES}

[1] Ubertini, P., Lebrun, F., Di Cocco, G., and et al., "IBIS: The Imager on-board INTEGRAL," Astron. \& Astrophys. 411, L131-L139 (2003).

[2] Frontera, F. and von Ballmoos, P., "Laue Gamma-Ray Lenses for Space Astrophysics: Status and Prospects," X-Ray Optics and Instrumentation, 2010. Special Issue on X-Ray Focusing: Techniques and Applications, id.215375 2010 (2010).

[3] Weidenspointner, G., Skinner, G., Jean, P., Knödlseder, J., von Ballmoos, P., Bignami, G., Diehl, R., Strong, A. W., Cordier, B., Schanne, S., and Winkler, C., "An asymmetric distribution of positrons in the Galactic disk revealed by $\gamma$-rays," Nature 451, 159-162 (Jan. 2008).

[4] Virgilli, E., Frontera, F., Valsan, V., Liccardo, V., Caroli, E., Stephen, J. B., Cassese, F., Recanatesi, L., Pecora, M., Mottini, S., Attiná, P., and Negri, B., "The LAUE project for broadband gamma-ray focusing lenses," in [Society of Photo-Optical Instrumentation Engineers (SPIE) Conference Series], Society of Photo-Optical Instrumentation Engineers (SPIE) Conference Series 8147 (Sept. 2011).

[5] Frontera, F., Loffredo, G., Pisa, A., Nobili, F., Carassiti, V., Evangelisti, F., Landi, L., Squerzanti, S., Caroli, E., Stephen, J. B., Andersen, K. H., Courtois, P., Auricchio, N., Milani, L., and Negri, B., "Focusing of gamma-rays with Laue lenses: first results," in [Society of Photo-Optical Instrumentation Engineers (SPIE) Conference Series], Society of Photo-Optical Instrumentation Engineers (SPIE) Conference Series $\mathbf{7 0 1 1}$ (Aug. 2008).

[6] Virgilli, E., Frontera, F., Valsan, V., Liccardo, V., Carassiti, V., Evangelisti, F., and Squerzanti, S., "Laue lenses for hard x-/soft $\gamma$-rays: new prototype results," in [Society of Photo-Optical Instrumentation Engineers (SPIE) Conference Series], Society of Photo-Optical Instrumentation Engineers (SPIE) Conference Series $\mathbf{8 1 4 7}$ (Sept. 2011). 
[7] Guidi, V., Barrière, N., Bellucci, V., Camattari, R., and Neri, I., "Bent crystals by surface grooving method for high-efficiency concentration of hard x-ray photons by a Laue lens," in [Society of Photo-Optical Instrumentation Engineers (SPIE) Conference Series], Society of Photo-Optical Instrumentation Engineers (SPIE) Conference Series $\mathbf{8 1 4 7}$ (Sept. 2011).

[8] Buffagni, E., Ferrari, C., Rossi, F., Marchini, L., and Zappettini, A., "Preparation of bent crystals as high-efficiency optical elements for hard x-ray astronomy," Optical Engineering 51, 056051 (2012).

[9] Valsan, V., Frontera, F., Virgilli, E., and Liccardo, E., "Expected performance of a Laue lens based on bent crystals," in [Society of Photo-Optical Instrumentation Engineers (SPIE) Conference Series], Society of Photo-Optical Instrumentation Engineers (SPIE) Conference Series $\mathbf{8 4 4 3}$ (2012).

[10] Liccardo, V., Virgilli, E., Frontera, F., and Valsan, V., "Characterization of bent crystals for Laue lenses," in [Society of Photo-Optical Instrumentation Engineers (SPIE) Conference Series], Society of Photo-Optical Instrumentation Engineers (SPIE) Conference Series 8443 (2012). 